\title{
Myasthenia gravis
}

\author{
Pierre R. Bourque MD, Ari Breiner MD
}

Cite as: CMAJ 2018 September 24;190:E1141. doi: 10.1503/cmaj.180656

\section{Myasthenia gravis causes localized muscle fatigability \\ 1 and weakness}

Although fatigue is a common medical complaint, fatigue related to myasthenia gravis has several key features: localized muscle weakness in a characteristic distribution, ${ }^{1}$ which is exacerbated by repeated activity and worsens late in the day. Asymmetric ptosis and variable diplopia are the presenting symptoms in $60 \%$ of patients, and, in most patients, it will spread to other muscles causing fluctuating dysarthria, dysphagia, difficulty chewing and dyspnea. Limb involvement is less common but typically results in proximal weakness. ${ }^{2}$

\section{2}

\section{Myasthenia gravis has two peaks of incidence}

Myasthenia gravis, an autoimmune disorder, is an uncommon condition, with a prevalence of about 30 cases per 100000 population. ${ }^{3}$ However, 20- to 40-year-old women and older men (who have a higher incidence of thymoma that is associated with myasthenia gravis) are most commonly affected. $^{3}$

\section{3}

\section{Myasthenia gravis affects only the skeletal muscles}

In a young patient with diplopia, the differential diagnosis should include multiple sclerosis. Additional sensory symptoms are common in multiple sclerosis but are always absent in myasthenia gravis. In older patients who present with dyspnea or limb weakness, amyotrophic lateral sclerosis should be considered; however, amyotrophic lateral sclerosis spares ocular movements and typically causes muscle fasciculations. ${ }^{1}$

\section{4}

\section{Simple clinical testing can be used to identify focal}

muscle fatigue

Tests include getting the patient to look upwards for one minute to observe for worsening ptosis, getting the patient to stare at an object laterally for 30-60 seconds and to report double vision if it occurs, and asking the patient to complete five consecutive resisted shoulder abductions to observe for gradual weakening of the deltoid muscles. ${ }^{1}$

\section{Electrophysiologic and serologic tests are used \\ for confirmation}

A neurologist typically carries out most investigations (Box 1). Singlefibre electromyography has the highest sensitivity (90\%). ${ }^{4}$ Tests for serum antibodies are highly specific but lack sensitivity when there is pure ocular involvement. ${ }^{4}$ Computed tomography of the chest is usually ordered to rule out thymoma once a diagnosis of myasthenia gravis has been confirmed.
Box 1: Laboratory investigations for myasthenia gravis ${ }^{4}$

Tests for serum antibodies

- Acetylcholine receptor antibodies are highly specific and positive in about $85 \%$ of cases of generalized ocular myasthenia gravis but only in $50 \%$ of pure ocular disease.

Electrodiagnostic testing

- Includes repetitive nerve stimulation testing (which has relatively poor sensitivity) and singlefibre electromyography (which has high sensitivity but must be performed by a neuromuscular specialist).

Tensilon test

- The administration of edrophonium intravenously (yielding transient improvement of weakness) is obsolete in North America because of drug shortages and concerns about cardiorespiratory compromise.

\section{Computed tomography of the chest}

- Performed after the diagnosis of myasthenia gravis to rule out the presence of thymoma (occurs in $10 \%-15 \%$ of patients).

\section{References}

1. Spillane J, Higham E, Kullman DM. Myasthenia gravis. BMJ 2012;345:e8497.

2. Gilhus NE. Myasthenia gravis (review article). N Engl J Med 2016;375:2570-81.

3. Breiner A, Widdifield J, Katzberg HD, et al. Epidemiology of myasthenia gravis in Ontario, Canada. Neuromuscul Disord 2016;26:41-6

4. Benatar M. A systematic review of diagnostic studies in myasthenia gravis. Neuromuscul Disord 2006;16:459-67.

\section{Competing interests: None declared.}

This article has been peer reviewed.

Affiliations: Division of Neurology, Department of Medicine (Bourque, Breiner), The Ottawa Hospital and University of Ottawa; Ottawa Hospital Research Institute (Bourque, Breiner), Ottawa, Ont.

Correspondence to: Pierre Bourque, pbourque@toh.ca 